\title{
Thirteen year retrospective review of the spectrum of inborn errors of metabolism presenting in a tertiary center in Saudi Arabia
}

\author{
Majid Alfadhel ${ }^{1,2,3,6^{*}}$ (D), Mohammed Benmeakel ${ }^{2}$, Mohammad Arif Hossain ${ }^{1,7}$, Fuad Al Mutairi ${ }^{1,2,3}$, Ali Al Othaim ${ }^{2,3,4}$, \\ Ahmed A. Alfares $s^{3,4,5}$, Mohammed Al Balwi $i^{2,3,4}$, Abdullah Alzaben ${ }^{1,2,3}$ and Wafaa Eyaid ${ }^{1,2,3}$
}

\begin{abstract}
Background: Inborn errors of metabolism (IEMs) are individually rare; however, they are collectively common. More than 600 human diseases caused by inborn errors of metabolism are now recognized, and this number is constantly increasing as new concepts and techniques become available for identifying biochemical phenotypes. The aim of this study was to determine the type and distribution of IEMs in patients presenting to a tertiary care center in Saudi Arabia. METHOD: We conducted a retrospective review of children diagnosed with IEMs presenting to the Pediatric Department of King Abdulaziz Medical City in Riyadh, Saudi Arabia over a 13-year period.

Results: Over the 13-year period of this retrospective cohort, the total number of live births reached 110,601. A total of 187 patients were diagnosed with IEMs, representing a incidence of 169 in 100,000 births (1:591). Of these, 121 patients (64.7\%) were identified to have small molecule diseases and 66 (35.3 \%) to have large molecule diseases. Organic acidemias were the most common small molecule IEMs, while lysosomal storage disorders (LSD) were the most common large molecule diseases. Sphingolipidosis were the most common LSD.

Conclusion: Our study confirms the previous results of the high rate of IEMs in Saudi Arabia and urges the health care strategists in the country to devise a long-term strategic plan, including an IEM national registry and a high school carrier screening program, for the prevention of such disorders. In addition, we identified 43 novel mutations that were not described previously, which will help in the molecular diagnosis of these disorders.
\end{abstract}

Keywords: Inborn errors of metabolism, IEMs, Saudi Arabia, Lysosomal, Organic acidemia, Mitochondrial, Fatty acid oxidation defects

\section{Background}

Inborn errors of metabolism (IEMs) are defined as monogenic diseases that result in dysfunctional proteins encoded by different genes, which in many cases lead to loss of activity of the enzymes involved [1]. More than 600 different inborn errors of metabolism have been recognized up to this point, and this number is constantly increasing as new concepts and techniques become available for identifying biochemical phenotypes. IEMs

\footnotetext{
* Correspondence: dralfadhelm@gmail.com

${ }^{1}$ Department of Pediatrics, King Abdulaziz Medical City, Riyadh, Saudi Arabia

${ }^{2}$ King Saud bin Abdulaziz University for Health Sciences, King Abdulaziz

Medical City, Riyadh, Saudi Arabia

Full list of author information is available at the end of the article
}

are extremely heterogeneous making their classification a primary challenge. Several informal systems of classification currently exist. IEMs can be classified according to the organs involved, such as neurological or hepatic disorders or according to the organelle involved, such as mitochondrial, peroxisomal or lysosomal disorders. Disorders can also be classified according to the age of presentation ranging from neonatal onset to juvenile and adult onset. Because each of these approaches may depend upon the actual setting, no single classification is universally applied [2]. One common and informative classification system involves the classification of IEMs into small and large molecule disorders [3]. Small 
molecule IEMs have an acute intoxication presentation with a remitting-relapsing clinical course. These include organic acidemias, vitamin responsive disorders, urea cycle disorders, inborn errors of carbohydrates, haem synthesis defects, cholesterol biosynthesis defects, and amino acids and metal transport defects. Large molecule IEMs have a gradual and insidious progressive presentation. These include glycogen storage disorders, sphingolipidosis, mucopolysaccharidosis, oligosaccharidosis, mitochondrial disorders and congenital disorders of glycosylation [2]. The diagnosis of IEMs is mainly based on biochemical investigations, which include the screening of several metabolites in the blood, urine and cerebrospinal fluid (CSF); analysis of enzymatic activities, and molecular genetics testing.

The incidence of such disorders vary from country to country and from region to region. In one Australian study the incidence was reported to be 15.7 per 100,000 births whereas, in Italy, the reported incidence was 27 per 100,000 births $[4,5]$. In the West Midlands region in the United Kingdom (UK), the incidence reached up to 1:784 and in British Columbia, Canada, the incidence was reported to be $1: 2500[6,7]$.

These disorders are usually inherited as autosomal recessive disorders, explaining why IEMs are common in populations with a high rate of consanguineous marriages, such as Saudi Arabia. In Saudi Arabia, the rate of consanguineous marriages reaches up to approximately $60 \%[8,9]$.

Despite the high frequency of IEMs, apart from the eastern region study [10], only a few anecdotal epidemiological studies in Saudi Arabia have discussed the incidence, type and distribution of such devastating disorders. Most of the remaining reports in the literature were limited to case reports and case series. In this study, we report the incidence, type, and distribution of IEMs presenting to King Abdulaziz Medical City (KAMC) in the middle region of Saudi Arabia over 13 years. In addition, we identified 43 novel mutations.

\section{Methods}

King Abdulaziz Medical City (KAMC) is a tertiary center in Saudi Arabia, that commenced operations in 1983. The bed capacity is 715 , and an average of 8500 births per year occur at the center. This medical city passed the requirements for accreditation under the Joint Commission International (JCI) standards with excellent performance in December 2006. The current study is a retrospective review of all cases at the Pediatric Department of King Abdulaziz Medical City (KAMC) in Riyadh, Saudi Arabia. The duration of the study was 13 years, from 01-01-2001 to 31-12-2014. All patients included were born during this period. The cases were identified according to medical records via diagnostic codes and the genetic division's database. The study was approved by the research committee of King Abdullah International Medical Research Centre (KAIMRC) in Riyadh, Saudi Arabia.

The diagnostic algorithm starts by referring patients based on clinical suspicion to genetic/metabolic facility from other departments, and from positive new born screening (NBS) after 2011. All accepted cases undergo phenotype related screening biochemical investigations. Confirmation of diagnosis is then achieved either by measuring enzyme activity and/or by targeted molecular tests. In case of unrevealing confirmatory investigations or vague presentation, whole exome or whole genome sequencing is requested.

The diagnosis of each IEM was based on clinical and biochemical investigations, including analyses of ammonia, lactic acid, total homocysteine, plasma amino acids, the acylcarnitine profile, urine aminoacids, urine for organic acids, copper, ceruloplasmine levels, very long chain fatty acids, transferrin isoelectrofocusing, carbohydrate-deficient transferrin and urine for polyols.

DNA molecular genetic testing was performed in commercial clinical international labs including CENTOGENE, GeneDx, Emory Genetics, Cincinnati Children's Hospital Medical Center, Bioscientia and Nijmegen Medical Center. All of the parents of the patients with IEMs were tested for carrier status.

Incidence was calculated by dividing the number of diagnosed cases by the number of total births during the study period and multiplying by 100,000 [11].

\section{Results}

Over the 13-year period of this retrospective cohort study, the total number of live births reached 110,601. A total of 187 patients were diagnosed with an IEM, resulting in an incidence of 169 in 100,000 births (1:591). Of these patients, $121(64.7 \%)$ were identified to have a small molecule disease (Table 1) and 66 (35.3\%) to have a large molecule disease (Table 2). The overall mean, median and range of age at diagnosis were 3.2 years, 1.2 years and from 1 day to 13 years respectively. Tables 1 and 2 show the type and distribution of IEMs from 2001 to 2014 and illustrate the estimated incidence per 100,000 live births for each group of disorders and the mean, median and range of age at diagnosis for each group. The lysosomal storage disorders (LSDs) were the most common diagnosed group, in general, and were observed in $39 / 187$ patients (20.8\%). Sphingolipidoses represent the largest subgroup of the LSDs (22/39; $56.4 \%$ ), and GM1 gangliosidosis (infantile phenotype) was the most prevalent disorder. The second most common category was organic acidemias (34/187; $18.2 \%)$, 
Table 1 Small-molecule disorders of IEMs in KAMC (2001-2014). Total numbers of live births $(110,601)$

\begin{tabular}{|c|c|c|c|c|c|}
\hline Disease category & $\begin{array}{l}\text { Number of cases } \\
\text { diagnosed }\end{array}$ & $\begin{array}{l}\text { Incidence per } \\
100,000\end{array}$ & $\begin{array}{l}\text { Mean age at } \\
\text { diagnosis }\end{array}$ & $\begin{array}{l}\text { Median age at } \\
\text { diagnosis }\end{array}$ & Range of age \\
\hline Organic acidemias & 34 & 30 & 1.8 years & 60 days & 1 day-10 years \\
\hline Propionic acidemia & 9 & & 30.2 days & 20 days & 1 day -6 months \\
\hline Methylmalonic acidemia & 7 & & & & \\
\hline Mutase deficiency & 5 & & & & \\
\hline Cobalamin A defect & 1 & & & & \\
\hline Cobalamin C defect & 1 & & & & \\
\hline Glutaric acidemia & 3 & & & & \\
\hline 3-hydroxy-3-methylglutaryl-CoA lyase deficiency & 4 & & & & \\
\hline 3-Methylcrotonylco A carboxylase deficiency & 3 & & & & \\
\hline Biotinidase deficiency & 3 & & & & \\
\hline 3-Methylglutaconic Aciduria Type III & 1 & & & & \\
\hline Ethylmalonic encephalopathy & 1 & & & & \\
\hline B-ketothiolase deficiency & 1 & & & & \\
\hline Isovaleric acidemia & 1 & & & & \\
\hline Malonic aciduria & 1 & & & & \\
\hline Aminoacidopathies & 30 & 27 & 3.3 years & 10.5 months & 1 day-13 years \\
\hline Homocystinuria & 14 & & 7 years & 7.5 years & \\
\hline - Classical & 11 & & & & \\
\hline - MTHFR deficiency & 2 & & & & \\
\hline - MAT deficiency & 1 & & & & \\
\hline PKU & 5 & & & & \\
\hline - Classical & 3 & & & & \\
\hline • Non-PKU hyperphenylalaninemia & 2 & & & & \\
\hline Biopterin Synthesis Defect PTPS deficiency & 4 & & & & \\
\hline MSUD & 5 & & & & \\
\hline Asparagine synthetase deficiency & 2 & & & & \\
\hline Vitamins responsive disorders & 18 & 16 & 5.7 years & 5.5 years & 6 months -10 years \\
\hline Biotin Thiamine Responsive Basal Ganglia Disease & 17 & & & & \\
\hline Pyridoxine-dependent epilepsy & 1 & & & & \\
\hline Inborn Errors of Carbohydrates & 12 & 11 & 3.1 years & 1.3 years & 1 week-7 years \\
\hline Galactosemia & 4 & & & & \\
\hline Transaldolase deficiency & 6 & & & & \\
\hline Hereditary fructose intolerance & 1 & & & & \\
\hline Fructose 1,6 bisphosphatase deficiency & 1 & & & & \\
\hline Urea Cycle Disorders & 12 & 11 & 12 days & 7 days & 1 day-30 days \\
\hline Argininosuccinic Aciduria & 8 & & & & \\
\hline Citrullinemia & 4 & & & & \\
\hline Fatty Acid Oxidation Defects & 5 & 4 & 1.4 years & 2 days & 2 days -7 years \\
\hline VLCAD deficiency & 3 & & 21 days & 2 days & 2 days -60 days \\
\hline MCAD deficiency & 1 & & 2 days & 2 days & 2 days \\
\hline Carnitine uptake defect & 1 & & 7 years & 7 years & 7 years \\
\hline Aminoacids transport defect & 5 & 4 & 10 years & 11 years & $6-13$ years \\
\hline Cystinuria & 5 & & & & \\
\hline
\end{tabular}


Table 1 Small-molecule disorders of IEMs in KAMC (2001-2014). Total numbers of live births (110,601) (Continued)

\begin{tabular}{|c|c|c|c|c|c|}
\hline Metal transport defect & 2 & 2 & 8.5 years & 8.5 years & $7-10$ years \\
\hline Wilson disease & 2 & & & & \\
\hline Disorders of Haem biosynthesis & 2 & 2 & 12.5 years & 12.5 years & $12-13$ years \\
\hline Acute intermittent porphyria & 2 & & & & \\
\hline Cholesterol biosynthesis defect & 1 & 1 & 1 year & 1 year & 1 year \\
\hline CHILD syndrome & 1 & & & & \\
\hline Total & 121 & 109 & 3.3 years & 9 months & 1 day -13 years \\
\hline
\end{tabular}

MTHFR methylenetetrahydrofolatereductase, MAT methionine adenosyltransferase, $P K U$ phenylketonuria, MSUD maple syrup urine disease, VLCAD very long-chain acyl-CoA dehydrogenase, MCAD medium-chain acyl-CoA dehydrogenase, CHILD Congenital hemidysplasia with ichthyosiform erythroderma and limb defects, PTPS 6-Pyruvoyl-Tetrahydropterin Synthase

with propionic acidemia (PA) as the most common disorder in that group. Aminoacidopathies were diagnosed in 30/187 patients $(16 \%)$. Fatty acid oxidation defects (FAOD) were diagnosed in $5 / 187$ patients $(2.7 \%)$, and the frequency of patients with urea cycle disorders (UCD) was 12/187 (6.4 \%). The most common FAOD was very long-chain acyl-CoA dehydrogenase deficiency (VLCAD), while argininosuccinic aciduria was the most common UCD. Mucopolysaccharidoses (MPS) were diagnosed in $15 / 187$ patients (8 \%), with MPS VI as the predominant type. Fourty-three novel mutations were identified, and missense mutations were the most common type of mutations (Tables 3 and 4). All the listed novel mutations fit with the clinical features of the disease, biochemical biomarkers support genotype phenotype correlation and they segregate well within the patients and family members.

\section{Discussion}

In this report, we describe the incidence of IEMs in a single tertiary center in the middle region of Saudi Arabia over more than a decade. We reported an incidence of 1:591 individuals, which is the highest incidence for IEMs reported to this point. Our study is the second epidemiological report after that of Moammar et al., 2010, who reported a incidence of 1:667 [10]. If we combine the two studies, the cumulative incidence is 1:635 births or 157 per 100,000, which is still one of highest reported incidence rates across the world. KAMC is one of the largest medical institutions in the Kingdom and is also a referral center; therefore to obtain a more accurate estimation of incidence of these disorders we have excluded the 69 patients who were diagnosed with IEM but not born at KAMC. If we combine the referred cases with those of patients born at the hospital, we obtain an incidence of 231.5 in 100,000 births (1:432).

Our study confirms the conclusions drawn by Moammar et al., 2010, LSDs are the most commonly identified group of disorders and organic acidemias are the most prevalent small molecule diseases. Our work also supports the notion that MPS VI is the most common type of mucopolysaccharidosis in Saudi Arabia. The results also support the wide variation in incidence of genetic diseases between Saudi Arabia and other parts of the world [12]. In our study for example the recorded number of PTPS exceeds the classical PKU which is reverse in Caucasian population [13]. This is more evident in PA, in which incidence in Saudi Arabia far exceeds the global incidence [14]. During the course of our study we discovered 9 new PA cases in our center, with incidence of 1 per 12,500 live births.

Our study showed unique phenotypes in comparison with the literature. Our three VLCAD patients for example had early presentation with severe phenotype ended with early death. Their genotype mutations were one novel missense mutation in exon 6, c.494 $\mathrm{T}>\mathrm{C}$ (p.Phe165Ser) and the other two had previously reported nonsense mutation in exon 2, c. $65 \mathrm{C}>\mathrm{A}$ (p.Ser22*). Although these mutations are not clear null mutations [15], they resulted in severe phenotype. Alternatively, the most common phenotype in VLCAD is the milder late onset with the missense mutation p.Val283Ala being the most prevalent disease causing variant [15]. This reflect the poor genotype-phenotype correlation.

In this study the number of private mutations was almost double that of founder mutations, which support the previous report of Al-Owain et.al (2012) who noted that private mutations outweigh founder mutations in Saudi Arabia [12].

Interestingly, among the diseases discovered by our study, 35 diseases are amenable for treatment. Recent advances in early diagnostic tools like the expanded New Born Screening program list, which can detect 14 of the listed diseases, and the availability of treatment options like enzyme replacement therapy, opened new horizons for these patients and their families.

With the implementation of next generation sequencing (WES and WGS) we were able to solve many obscure cases. Additionally, new diseases and new variants might be discovered more easily. It is possible to see NGS a first line diagnostic tool in the near future. 
Table 2 Large-molecule disorders of IEMs in KAMC (2001-2014). Total numbers of live births (110,601)

\begin{tabular}{|c|c|c|c|c|c|}
\hline Disease category & $\begin{array}{l}\text { Number of cases } \\
\text { diagnosed }\end{array}$ & $\begin{array}{l}\text { Incidence per } \\
100,000\end{array}$ & $\begin{array}{l}\text { Mean age at } \\
\text { diagnosis }\end{array}$ & $\begin{array}{l}\text { Median age at } \\
\text { diagnosis }\end{array}$ & Range of age \\
\hline Lysosomal Storage Diseases (LSD) & 41 & 37 & 3.6 year & 3 years & 2 months -13 years \\
\hline Sphingolipidosis & 22 & 20 & 3.1 years & 2 years & 2 months -13 years \\
\hline Fabry disease & 3 & & & & \\
\hline Sandhoff disease & 2 & & & & \\
\hline Niemann-Pick disease type B & 1 & & & & \\
\hline Niemann-Pick disease type $C$ & 3 & & & & \\
\hline GM1 gangliosidosis (infantile phenotype) & 4 & & & & \\
\hline Metachromatic leukodystrophy & 3 & & & & \\
\hline Saposin B Deficiency & 2 & & & & \\
\hline Krabbe disease & 1 & & & & \\
\hline Mucopolysaccharidosis (MPS) & 15 & 14 & 5 years & 5 years & 5 months -12 years \\
\hline MPS I & 1 & & & & \\
\hline MPS II & 1 & & & & \\
\hline MPS IIIA & 2 & & & & \\
\hline MPS IVA & 5 & & & & \\
\hline MPS VI & 6 & & & & \\
\hline Oligosaccharidosis & 2 & 2 & 3 years & 3 years & $2-4$ years \\
\hline Mucolipidosis II & 1 & & & & \\
\hline a-mannosidosis & 1 & & & & \\
\hline \multicolumn{6}{|l|}{ Others } \\
\hline Neuronal ceroid-lipofuscinoses & 3: 2 type 6 , and 1 type 8 & & 5.3 & 5 & $5-6$ years \\
\hline GSD \| & 2 & & 3.1 months & 3.1 months & 1 week to 6 months \\
\hline Glycogen storage diseases (GSD) & 5 & 4 & 2.2 years & 2 years & 15 months -4 years \\
\hline GSD III & 1 & & & & \\
\hline GSD IV & 1 & & & & \\
\hline GSD IX & 3 & & & & \\
\hline Mitochondrial disorders & 12 & 11 & 2.2 years & 8 months & 1 week -8 years \\
\hline Leigh disease & 3 & & & & \\
\hline Pyruvate dehydrogenase deficiency & 2 & & & & \\
\hline Pyruvate carboxylase deficiency & 2 & & & & \\
\hline Mitochondrial DNA depletion syndrome 3 & 1 & & & & \\
\hline Mitochondrial DNA depletion syndrome 5 & 1 & & & & \\
\hline $\begin{array}{l}\text { Cardioencephalomyopathy, fatal infantile, } \\
\text { due to cytochrome c oxidase deficiency } 1\end{array}$ & 1 & & & & \\
\hline $\begin{array}{l}\text { 3-Methylglutaconic aciduria with deafness, } \\
\text { encephalopathy, and Leigh-like }\end{array}$ & 1 & & & & \\
\hline Primary Coenzyme Q10 deficiency type 5 & 1 & & & & \\
\hline Peroxisomal disorders & 7 & 6 & 2 years & 9 months & 1 week-8 years \\
\hline Primary hyperoxaluria type 1 & 5 & & & & \\
\hline Zellweger syndrome & 1 & & & & \\
\hline Rhizomelic Chondrodysplasia Punctata & 1 & & & & \\
\hline Congenital disorders of glycosylation (CDG) & $1(\mathrm{CDG} 1 \mathrm{~L})$ & 1 & 8 years & 8 years & 8 years \\
\hline Total & 66 & 60 & 3.1 years & 2 years & 1 week-13 years \\
\hline
\end{tabular}


Table 3 Mutations for small molecule IEMs

\begin{tabular}{|c|c|c|c|c|c|c|}
\hline Disease category & Disease & Gene & Reported mutations & Novel mutations & $\begin{array}{l}\text { Founder Vs. } \\
\text { Private }\end{array}$ & Type of mutation \\
\hline \multirow[t]{19}{*}{ Organic acidemias } & Propionic acidemia & $P C C A$ & & c.425G > A(p. Gly142Asp) & Founder & Homozygous, missense \\
\hline & & & c.350G > A (p.Gly117Asp) & & Private & \\
\hline & & $P C C B$ & c.1050dupT & & Private & Dupplication \\
\hline & Methylmalonic acidemia & MUT & & c.329 A > G(p. Tyr110Cys) & Founder & Homozygous, missense, \\
\hline & & & c.1677-1G > C & & Private & Splice \\
\hline & Cobalamin A Defect & MMAA & c.586C > T (p.Arg196*) & & Private & Nonsense \\
\hline & Cobalamin C defect & MMACHC & c.394C > T (p. Arg132*) & & Private & Nonsense \\
\hline & Glutaric acidemia & $\mathrm{GCDH}$ & c.1144G > A (p.Ala382Thr) & & Private & missense \\
\hline & & & & c. $853-2 A>G($ IVS8-2A $>$ G) & Private & Splice \\
\hline & & & & c.278A > G (p.His93Arg) & Private & missense \\
\hline & $\begin{array}{l}\text { 3-hydroxy-3-methylglutaryl-CoA lyase } \\
\text { deficiency }\end{array}$ & $H M G C L$ & c. $122 \mathrm{G}>\mathrm{A}$ & & Founder & missense \\
\hline & $\begin{array}{l}\text { 3-Methylcrotonyl CoA carboxylase } \\
\text { deficiency }\end{array}$ & MCCC1 & & $\begin{array}{l}\text { c. } 1808 \text { dup A(p. p.Asn603 } \\
\text { Lysfs*5) }\end{array}$ & Private & Homozygous, duplication \\
\hline & & MCCC2 & c.1147A > T (p.Lys383*) & & Private & Nonsense \\
\hline & Biotinidase deficiency & BTD & $\begin{array}{l}\text { c.755A > G (p.Asp252Gly) } \\
\text { c.1330G > C (p.Asp444His) }\end{array}$ & & Private & $\begin{array}{l}\text { Two heterozygous missense } \\
\text { mutations in Exon } 4\end{array}$ \\
\hline & 3-Methylglutaconic aciduria type III & OPA3 & & c.194delG(p. Gly65Alafs*7) & Private & Homozygous, deletion \\
\hline & Ethylmalonic encephalopathy & ETHE1 & & c.263 C> T(p. Ser88Leu) & Private & Homozygous, missense \\
\hline & B-Ketothiolase deficiency & ACAT1 & & $\begin{array}{l}\text { c.412-419del(p. } \\
\text { Gln138Tyrfs*36) }\end{array}$ & Private & Homozygous, deletion \\
\hline & Isovaleric acidemia & IVD & & c.358C > T(p. Arg120X) & Private & Homozygous, nonsense \\
\hline & Malonyl-CoA decarboxylase deficiency & MLYCD & & $\begin{array}{l}\text { c.953_954delAG(p. } \\
\text { Glu318Valfs*35) }\end{array}$ & Private & Homozygous, deletion \\
\hline \multirow[t]{10}{*}{ Aminoacidopathies } & Homocystinuria & & & & & \\
\hline & Classical & CBS & c.969G > A (p.Trp323Ter) & & Founder & Homozygous missense \\
\hline & & & c.1006C > T (p.Arg336Cys) & & Founder & Homozygous missense \\
\hline & MTHFR deficiency & MTHFR & c.680C > T (p.Thr227Met) & & Private & \\
\hline & MAT deficiency & MATTA & & c.1081G > T(p.Val361Phe) & Private & Homozygous, missense \\
\hline & PKU & PAH & c.1169A > G (p.Glu390Gly) & & Private & Homozygous, missense \\
\hline & PTPS deficiency & PTPS & & c.238A > G(p. Met80Val) & Founder & Homozygous, missense \\
\hline & & & c.169_171delGTG (p.Val57del) & & Founder & Homozygous, deletion \\
\hline & MSUD & $B C K D H A$ & & c.347A > G(p. Asp116Gly) & Private & missense \\
\hline & & & c.905A > C (p.Asp302Ala) & & Founder & missense \\
\hline
\end{tabular}


Table 3 Mutations for small molecule IEMs (Continued)

\begin{tabular}{|c|c|c|c|c|c|c|}
\hline & & \multicolumn{2}{|l|}{$B C K D H B$} & c.674 T > C(p.Leu225Pro) & Private & missense \\
\hline & Asparagine synthetase deficiency & ASNS & & c.1160A > G (p.Tyr377Cys) & Founder & Homozygous, missense \\
\hline \multirow[t]{2}{*}{$\begin{array}{l}\text { Vitamins responsive } \\
\text { disorders }\end{array}$} & $\begin{array}{l}\text { Biotin Thiamine Responsive Basal Ganglia } \\
\text { Disease }\end{array}$ & SLC19A3 & c.1264A > G (p.Thr422Ala) & & Founder & Homozygous, missense \\
\hline & Pyridoxine-dependent epilepsy & ALDHTAl & c.877dupAA (p.Ser293Lysfs*22) & & Private & Duplication \\
\hline \multirow{6}{*}{$\begin{array}{l}\text { Inborn Errors of } \\
\text { Carbohydrates }\end{array}$} & Galactosemia & GALT & c.691 C > T (p.Arg231Cys) & & Founder & Homozygous, missense \\
\hline & & & c.404C > T (p.Ser135Leu) & & Private & Homozygous, missense \\
\hline & & & C.563A > G (P.Gln188Arg) & & Private & Homozygous, missense \\
\hline & Transaldolase Deficiency & TALDO1 & c.793delC (p.Gln265ArgfsX56) & & Founder & Deletion \\
\hline & Hereditary fructose intolerance & $A L D O B$ & $\begin{array}{l}\text { c.360_363delCAAA } \\
\text { (p.Asn119LysfsX31) }\end{array}$ & & Private & Deletion \\
\hline & Fructose 1,6 bisphosphatase deficiency & $F B P 1$ & $\begin{array}{l}\text { c.114_119dup (p. } \\
\text { Cys39_Thr40dup) }\end{array}$ & & Private & Duplication \\
\hline \multirow[t]{4}{*}{ Urea cycle disorders } & Argininosuccinic Aciduria & ASL & c.556C > T (p.Arg186Trp) & & Founder & Missense \\
\hline & & & $c .1060 C>T(p . Q 354 X)$ & & Founder & Nonsense \\
\hline & Citrullinemia type 1 & ASS1 & & c. $364-2 A>G$ & Founder & Homozygous, intronic \\
\hline & & & c.370G > A (p.Asp124Asn) & & Founder & Homozygous, missense \\
\hline \multirow[t]{5}{*}{ Fatty acid oxidation defect } & VLCAD & ACADVL & & c.494 T > C(Phe165Ser) & Private & Homozygous, missense \\
\hline & VLCAD & ACADVL & c. $65 \mathrm{C}>\mathrm{A}\left(\mathrm{p} . \mathrm{Ser} 22^{*}\right)$ & & Founder & Nonsense \\
\hline & MCAD & ACADM & & c.255 G > T(p.Gly119*); & Private & Homozygous, nonsense \\
\hline & & & & c.938 T > G(p.Phe313Cys & Private & Homozygous, missense \\
\hline & Carnitine uptake defect & SLC22A5 & & c.1385G > A(p. Gly462Asp) & Private & Homozygous, missense \\
\hline \multirow{3}{*}{$\begin{array}{l}\text { Aminoacids transport } \\
\text { defect }\end{array}$} & Cystinuria & $S L C 3 A 1$ & & c.1711 T> A(p.Cys571Ser) & Founder & Homozygous, missense \\
\hline & & & c.1400 T > A (p.Met467Lys) & & Private & \\
\hline & & SLC7A9 & & c.1166 C > T(p.Thr389Met) & Private & Homozygous, missense \\
\hline Metal Transport Defect & Wilson disease & $A T P 7 B$ & c.2230 T > C (p.Ser744Pro) & & Founder & Homozygous, missense \\
\hline $\begin{array}{l}\text { Disorders od Haem } \\
\text { biosynthesis }\end{array}$ & Acute Intermittent Poephyria & HMBS & c.760delC (p.Leu254X) & & Founder & Nonsense \\
\hline $\begin{array}{l}\text { Cholesterol biosynthesis } \\
\text { defect }\end{array}$ & CHILD syndrome & NSDHL & c.314C > T (p.Ala105Val) & & Private & Homozygous, missense \\
\hline
\end{tabular}

PKU phenylketonuria, MSUD maple syrup urine disease, VLCAD very long-chain acyl-CoA dehydrogenase, MCAD medium-chain acyl-CoA dehydrogenase 
Table 4 Mutations for large molecule IEMs

\begin{tabular}{|c|c|c|c|c|c|c|c|}
\hline \multicolumn{2}{|c|}{ Disease category } & \multirow{2}{*}{$\begin{array}{l}\text { Disease } \\
\text { Fabry }\end{array}$} & \multirow{2}{*}{$\begin{array}{l}\text { Gene } \\
\text { GLA }\end{array}$} & \multirow{2}{*}{$\begin{array}{l}\text { Reported mutations } \\
\text { c. } 782 \mathrm{G}>\mathrm{T} \text { (p.Gly261Val) }\end{array}$} & \multirow[t]{2}{*}{ Novel mutations } & \multirow{2}{*}{$\begin{array}{l}\text { Founder Vs. } \\
\text { Private } \\
\text { Founder }\end{array}$} & \multirow{2}{*}{$\begin{array}{l}\text { Type of mutation } \\
\begin{array}{l}\text { Homozygous, } \\
\text { missense }\end{array}\end{array}$} \\
\hline$\overline{\mathrm{LSD}}$ & Sphingolipidosis & & & & & & \\
\hline & & Sandhoff disease & HEXB & & $\begin{array}{l}\text { c.1169+3_1169+10delAAGTTGTT } \\
\text { (p.Gly65 AlafsX7) }\end{array}$ & Private & Deletion \\
\hline & & Niemann-Pick disease type B & SMPD1 & c.1267 C > T (p.His423Tyr) & & Founder & $\begin{array}{l}\text { Homozygous, } \\
\text { missense }\end{array}$ \\
\hline & & \multirow[t]{2}{*}{ Niemann-Pick disease type $C$} & \multirow[t]{2}{*}{$N P C 1$} & & c. $2130+1 G>A$ & Founder & $\begin{array}{l}\text { Homozygous, } \\
\text { intronic }\end{array}$ \\
\hline & & & & & c.2443_2444delp.ser815Leufs*54 & Private & deletion \\
\hline & & \multirow[t]{2}{*}{ GM1 gangliosidosis } & \multirow[t]{2}{*}{ GLB1 } & & c.950G > A(p. Trp317*) & Private & $\begin{array}{l}\text { Homozygous, } \\
\text { nonsense }\end{array}$ \\
\hline & & & & c.171C > G (p.Tyr57X) & & Founder & $\begin{array}{l}\text { Homozygous, } \\
\text { missense }\end{array}$ \\
\hline & & Metachromatic leukodystrophy & ARSA & & c. $1108-2 A>G$ & Private & $\begin{array}{l}\text { Homozygous, } \\
\text { intronic }\end{array}$ \\
\hline & & Saposin B deficiency & PSAP & c.722G > C (p.Cys241Ser & & Founder & $\begin{array}{l}\text { Homozygous, } \\
\text { missense }\end{array}$ \\
\hline & & Krabbe disease & GALC & & c.396G > A(p.Trp132*) & Private & $\begin{array}{l}\text { Homozygous, } \\
\text { nonsense }\end{array}$ \\
\hline \multirow{10}{*}{\multicolumn{2}{|c|}{$\begin{array}{l}\text { Mucopolysaccharidosis } \\
\text { (MPS) }\end{array}$}} & MPSI & IDUA & & c.1868 T > C(p. Leu623Pro) & Private & $\begin{array}{l}\text { Homozygous, } \\
\text { missense }\end{array}$ \\
\hline & & MPSII & IDS & & c.405A > C(p. Lys135Asn) & Private & $\begin{array}{l}\text { Homozygous, } \\
\text { missense }\end{array}$ \\
\hline & & \multirow[t]{2}{*}{ MPSIIIA } & \multirow[t]{2}{*}{ SGSH } & & c. $664-13 C>G$ & Private & $\begin{array}{l}\text { Homozygous, } \\
\text { intronic }\end{array}$ \\
\hline & & & & c.535G > A (p.Asp179Asn) & & Private & $\begin{array}{l}\text { Homozygous, } \\
\text { missense }\end{array}$ \\
\hline & & \multirow[t]{3}{*}{ MPS IVA } & \multirow[t]{3}{*}{ GALNS } & c. $120+1 G>C($ IVS1 + 1G >C) & & Private & $\begin{array}{l}\text { Homozygous, } \\
\text { missense }\end{array}$ \\
\hline & & & & c.860C > T (p.Ser287Leu) & & Private & $\begin{array}{l}\text { Homozygous, } \\
\text { missense }\end{array}$ \\
\hline & & & & c.697G > A (p.Asp233Asn) & & Private & $\begin{array}{l}\text { Homozygous, } \\
\text { missense }\end{array}$ \\
\hline & & \multirow[t]{3}{*}{ MPSVI } & \multirow[t]{3}{*}{$A R S B$} & c.753C > G (p.Tyr251*) & & Founder & $\begin{array}{l}\text { Homozygous, } \\
\text { nonsense }\end{array}$ \\
\hline & & & & c.430A > G (p.His393ARG) & & Founder & $\begin{array}{l}\text { Homozygous, } \\
\text { missense }\end{array}$ \\
\hline & & & & c.1079 T > C (p. Leu360Pro) & & Private & $\begin{array}{l}\text { Homozygous, } \\
\text { missense }\end{array}$ \\
\hline
\end{tabular}


Table 4 Mutations for large molecule IEMs (Continued)

\begin{tabular}{|c|c|c|c|c|c|c|}
\hline \multirow[t]{2}{*}{ Oligosaccharidosis } & Mucolipidosis II & GNPTAB & $\begin{array}{l}\text { c.3503_3504 delTC } \\
\text { (p.Phex1172) }\end{array}$ & & Private & $\begin{array}{l}\text { Homozygous, } \\
\text { deletion }\end{array}$ \\
\hline & a-mannosidosis & MAN2B1 & c.1340A > T (p.Asp447Val) & & Private & $\begin{array}{l}\text { Homozygous, } \\
\text { missense }\end{array}$ \\
\hline \multirow[t]{5}{*}{ Others } & NCL type 6 & CLN6 & & c.794_796del(p.Ser265del) & Private & $\begin{array}{l}\text { Homozygous, } \\
\text { deletion }\end{array}$ \\
\hline & & & c.794_796delCCT & & Private & $\begin{array}{l}\text { Homozygous, } \\
\text { deletion }\end{array}$ \\
\hline & NCL type 8 & CLN8 & $\begin{array}{l}\text { Homozygous deletion } \\
\text { encompassing exon2 }\end{array}$ & & Private & $\begin{array}{l}\text { Homozygous, } \\
\text { deletion }\end{array}$ \\
\hline & GSDII & GAA & & c.1431delT(p. Ile477fs) & Private & $\begin{array}{l}\text { Homozygous, } \\
\text { deletion }\end{array}$ \\
\hline & & & & c. 1657 C > T(p. Gln553*) & Private & $\begin{array}{l}\text { Homozygous } \\
\text { nonsense }\end{array}$ \\
\hline \multirow[t]{4}{*}{ Slycogen storage disease } & GSDIII & $A G L$ & & c.4353G > T(p. Trp1451Cys); & Private & $\begin{array}{l}\text { Homozygous, } \\
\text { missense }\end{array}$ \\
\hline & GSDIV & GBE1 & & c.998A > T (p.Glu 333 Val) & Private & $\begin{array}{l}\text { Homozygous, } \\
\text { missense }\end{array}$ \\
\hline & GSD IX & PHKG2 & c. $130 \mathrm{C}>\mathrm{T}\left(\mathrm{p} . \operatorname{Arg} 44^{*}\right)$ & & Founder & $\begin{array}{l}\text { Homozygous } \\
\text { nonsense }\end{array}$ \\
\hline & & PHKB & & Deletion Exon 5 and 6 & Private & $\begin{array}{l}\text { Homozygous, } \\
\text { deletion }\end{array}$ \\
\hline \multirow[t]{10}{*}{ Mitochondrial disorders } & Leigh disease & MTATP6 & m.8993 T > G (p.Leu156Arg) & & Private & $\begin{array}{l}\text { Homoplasmic, } \\
\text { missense }\end{array}$ \\
\hline & & $\operatorname{cox} 15$ & c.649C > T (p.Arg217Trp) & & Private & $\begin{array}{l}\text { Homozygous, } \\
\text { missense }\end{array}$ \\
\hline & Pyruvate dehydrogenase deficiency & PDHA1 & $\begin{array}{l}\text { c.1256_1259dup } \\
\text { (p.Trp421Serfs*6) }\end{array}$ & & Private & $\begin{array}{l}\text { Heterozygous } \\
\text { Duplication }\end{array}$ \\
\hline & & PDHA1 & & c.1132C > T (p.Arg378Cys) & Private & $\begin{array}{l}\text { Hemizygous } \\
\text { missense }\end{array}$ \\
\hline & Pyruvate Carboxylase Deficiency & $P C$ & & c.3116_3126del (p.Leu1039GInfs*7) & Private & Deletion \\
\hline & Mitochondrial DNA depletion syndrome 3 & DGUOK & c. $617 G>$ A (p. R206k) & & Private & $\begin{array}{l}\text { Homozygous, } \\
\text { missense }\end{array}$ \\
\hline & Mitochondrial DNA depletion syndrome 5 & SUCLA2 & & c.362_363del (p.lle121Serfs*38) & Private & Deletion \\
\hline & $\begin{array}{l}\text { Cardioencephalomyopathy, fatal infantile, due to } \\
\text { cytochrome c oxidase deficiency } 1\end{array}$ & SCO2 & c.2 T > C(p.Met1?) & & Private & $\begin{array}{l}\text { Homozygous, } \\
\text { missense }\end{array}$ \\
\hline & $\begin{array}{l}\text { 3-Methylglutaconic aciduria with deafness, } \\
\text { encephalopathy, and Leigh-like }\end{array}$ & SERAC1 & c.438del (p.Thr147Argfs*22) & & Private & Deletion \\
\hline & Primary CoenzymeQ10 deficiency type 5 & COQ9 & & chr16_57485062C > T (p.His62Arg) & Private & $\begin{array}{l}\text { Homozygous, } \\
\text { missense }\end{array}$ \\
\hline
\end{tabular}


Table 4 Mutations for large molecule IEMs (Continued)

Peroxisomal disorders $\quad$ Primary hyperoxaluria type 1

AGXT

c.187G > C (p.Gly63Arg)

PEX5

c.1578 T > G (p.Asn526Lys)

Founder

Homozygous,

missense

Zellweger syndrome

PEX7

c.321_322delTA(p.Tyr107*)

Private

Homozygous,

Rhizomelic chondrodysplasia punctata type 1

ALG9

c.1075G > A (p.E359K)

missense

Congenital disorder of

CGD $1 \mathrm{~L}$

Private

deletion

glycosylation (CDG)

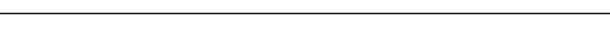


The markedly high numbers of metabolic diseases in Saudi Arabia in general and in our center in particular, with mostly homogenous genotypes, paves the way for future collaboration with international parties, research centers and drug industry, to help providing treatment for our patients. This environment is ideal for wide spectrum of clinical trials of various phases, to speed up the process of new medications discovery.

The limitations of the presented cohort study are clear. These include the fact that the study is confined to a single center in one particular region and contains a small sample size; therefore, the internal and external validity of the study is threatened. Such a retrospective review increases the risk of selection and information biases. Therefore, the numbers mentioned in this study should be taken with caution until further larger studies confirm or refute such findings. The retrospective nature risks missing cases due to poor documentation. In addition, some patients with metabolic disorders may not be seen at the medical center due to early death prior metabolic intervention. In addition, cases with a relatively mild disease may never have presented to the specialized metabolic center, which also contributes to the bias inherent in this study. The variability in ages at diagnosis is attributed to the delay in referring cases to the metabolic facility from other departments, and these numbers should not reflect the expected age of presentation for the listed diseases.

The incredibly high rate of IEMs in Saudi Arabia compels the health care administration in the country to develop a long-term strategic plan for the prevention of such disorders. First, a national registry should be implemented, and through that registry, a determination of the most common IEM and most common mutations in the population can be made. Second, genetic screening of high school students by DNA molecular testing should be performed to identify carriers for the most common disorders in the Saudi population. Premarital molecular screening can help couples, who carry the same disease causing variants, to take informed decision regarding their marriage and the consequences of their decision. Such a strategy has proven to be effective in another population [16]. Finally, intensive educational campaigns aimed at the community through schools, $\mathrm{TV}$, and web-based social media should be initiated.

\section{Conclusion}

In this study, we report the incidence, type, and distribution of IEMs presenting to King Abdulaziz Medical City (KAMC) in the middle region of Saudi Arabia over 13 years. We also identified 43 novel mutations in 37 genes. Our study emphasizes the high incidence of IEMs in the Saudi population and urges the health care administration in the country to develop a long-term strategic plan for the prevention of such disorders, including an IEMs national registry and a high school carrier screening program.

\section{Abbreviations}

CHILD: Congenital hemidysplasia with ichthyosiform erythroderma and limb defects; CSF: Cerebrospinal fluid; IEM: Inborn error of metabolism;

IEMs: Inborn errors of metabolism; IRB: Institutional review board; JCI: Joint Commission International; KAIMRC: King Abdullah International Medical

Research Centre; KAMC: King Abdulaziz Medical City; LSD: Lysosomal storage disorders; MAT: Methionine adenosyltransferase; MCAD: Medium-chain acyl-CoA dehydrogenase; MSUD: Maple syrup urine disease;

MTHFR: Methylenetetrahydrofolate reductase; PKU: Phenylketonuria;

SCAD: Small-chain acyl-CoA dehydrogenase; UCD: Urea cycle disorders; UK: United Kingdom; VLCAD: Very long-chain acyl-CoA dehydrogenase

\section{Acknowledgements}

We would like to thank Prof. Dr. Ronald J.A. Wanders from Academic Medical Center, University of Amsterdam for his valuable contribution.

\section{Funding}

This research received no specific grant from any funding agency in the public, commercial or not-for-profit sectors'.

\section{Availability of data and materials}

Not applicable, as this study is a retrospective chart review over a long period and the data are scattered in several files. Any data will be available upon the request of the reviewers.

\section{Authors' contributions}

MAF: performed the majority of the work associated with preparing, writing and submitting the manuscript and contributed to the clinical diagnosis and management of the patients from King Abdulaziz Medical City. MBM and $\mathrm{MAH}$ : summarized the clinical and molecular genetic data and contributed to the analysis part of the manuscript. AAO, AAF, MAB: edited the manuscript and contributed to the biochemical and molecular diagnosis of the patients summarized in the article. AAZ and WE: edited the manuscript. All of the authors read and approved the final manuscript.

\section{Competing interests}

The authors declare that they have no competing interests.

\section{Consent for publication}

Informed consent to participate in the study was obtained from the parents of participants.

\section{Ethics approval and consent to participate}

The study was approved by the research committee of King Abdullah International Medical Research Centre (KAIMRC) in Riyadh, Saudi Arabia.

\section{Author details}

${ }^{1}$ Department of Pediatrics, King Abdulaziz Medical City, Riyadh, Saudi Arabia. ${ }^{2}$ King Saud bin Abdulaziz University for Health Sciences, King Abdulaziz Medical City, Riyadh, Saudi Arabia. ${ }^{3}$ Ministry of National Guard-Health Affairs (NGHA), Riyadh, Saudi Arabia. ${ }^{4}$ Department of Pathology, King Abdulaziz Medical City, Riyadh, Saudi Arabia. ${ }^{5}$ Qassim University, College of Medicine, Department of Pediatrics, Almulyda, Saudi Arabia. ${ }^{6}$ Division of Genetics, Department of Pediatrics King Saud bin Abdulaziz University for Health Sciences, King Abdulaziz Medical City, RiyadhPO Box 2249011426, Saudi Arabia. ${ }^{7}$ Advanced Clinical Research Center, Shin-Yurigaoka General Hospital, Kawasaki, Kanagawa, Japan.

Received: 3 July 2016 Accepted: 6 September 2016

Published online: 15 September 2016

\section{References}

1. Scriver CR, Valle D, Beaudet L, Vogelstein B, Kinzler KW, Antonarakis SE, et al. The Online Metabolic and Molecular Bases of Inherited Disease. New York: McGraw-Hill Education.

2. Lanpher B, Brunetti-Pierri N, Lee B. Inborn errors of metabolism: the flux from Mendelian to complex diseases. Nat Rev Genet. 2006;7:449-60. 
3. Applegarth DA, Dimmick JE, Toone JR. Laboratory detection of metabolic disease. Pediatr Clin North Am. 1989;36:49-65.

4. Wilcken B, Wiley V, Hammond J, Carpenter K. Screening newborns for inborn errors of metabolism by tandem mass spectrometry. N Engl J Med. 2003;348:2304-12.

5. Dionisi-Vici C, Rizzo C, Burlina AB, Caruso U, Sabetta G, Uziel G, et al. Inborn errors of metabolism in the Italian pediatric population: a national retrospective survey. J Pediatr. 2002;140:321-7.

6. Sanderson S, Green A, Preece MA, Burton H. The incidence of inherited metabolic disorders in the West Midlands, UK. Arch Dis Child. 2006;91:896-9.

7. Applegarth DA, Toone JR, Lowry RB. Incidence of inborn errors of metabolism in British Columbia, 1969-1996. Pediatrics. 2000;105:e10

8. El-Hazmi MA, Al-Swailem AR, Warsy AS, Al-Swailem AM, Sulaimani R, Al-Meshari AA. Consanguinity among the Saudi Arabian population. J Med Genet. 1995; 32:623-6.

9. El Mouzan Ml, Al Salloum AA, Al Herbish AS, Qurachi MM, Al Omar AA. Consanguinity and major genetic disorders in Saudi children: a communitybased cross-sectional study. Ann Saudi Med. 2008;28:169-73.

10. Moammar H, Cheriyan G, Mathew R, Al-Sannaa N. Incidence and patterns of inborn errors of metabolism in the Eastern Province of Saudi Arabia, 1983-2008. Ann Saudi Med. 2010;30:271-7.

11. Elston RC. Introduction and overview. Statistical methods in genetic epidemiology. Stat Methods Med Res. 2000;9:527-41

12. Al-Owain M, Al-Zaidan H, Al-Hassnan Z. Map of autosomal recessive genetic disorders in Saudi Arabia: concepts and future directions. Am J Med Genet A. 2012:158A:2629-40.

13. Al Aqeel A, Ozand PT, Gascon G, Nester M, Al Nasser M, Brismar J, et al. Biopterin-dependent hyperphenylalaninemia due to deficiency of 6-pyruvoyl tetrahydropterin synthase. Neurology. 1991;41:730-7.

14. Zayed H. Propionic acidemia in the Arab World. Gene. 2015;564:119-24.

15. Andresen BS, Olpin S, Poorthuis BJ, Scholte HR, Vianey-Saban C, Wanders $\mathrm{R}$, et al. Clear correlation of genotype with disease phenotype in very-long-chain acyl-CoA dehydrogenase deficiency. Am J Hum Genet. 1999;64:479-94.

16. Mitchell JJ, Capua A, Clow C, Scriver CR. Twenty-year outcome analysis of genetic screening programs for Tay-Sachs and beta-thalassemia disease carriers in high schools. Am J Hum Genet. 1996;59:793-8,

\section{Submit your next manuscript to BioMed Central and we will help you at every step:}

- We accept pre-submission inquiries

- Our selector tool helps you to find the most relevant journal

- We provide round the clock customer support

- Convenient online submission

- Thorough peer review

- Inclusion in PubMed and all major indexing services

- Maximum visibility for your research

Submit your manuscript at www biomedcentral.com/submit
() BioMed Central 